Richard P. Allen, PhD

Peter B. Barker, DPhil Alena Horská, PhD

Christopher J. Earley, $\mathrm{MD}, \mathrm{PhD}$

Correspondence to

Dr. Allen:

richardjhu@mac.com

\title{
Thalamic glutamate/glutamine in restless legs syndrome
}

\author{
Increased and related to disturbed sleep
}

\section{ABSTRACT}

Objective: To evaluate possible abnormal increase in thalamic glutamate/glutamine levels for restless legs syndrome (RLS) indicating increased glutamatergic activity producing arousal that at night disrupts and shortens sleep.

Methods: ${ }^{1 H}$ MRS of the right thalamus was performed using a $1.5 \mathrm{~T}$ GE MRI scanner and the PROBE-P (PRESS) on 28 patients with RLS and 20 matched controls. The Glx signal (combination of mostly glutamate $[\mathrm{Glu}]$ and glutamine $[\mathrm{GIn}]$ ) was assessed as a ratio to the total creatine (Cr). This study tested 2 primary hypotheses: 1 ) higher thalamic $\mathrm{G} / x / \mathrm{Cr}$ for patients with RLS than controls; 2) thalamic Glx/Cr correlates with increased wake during the sleep period.

Results: The GIx/Cr was higher for patients with RLS than controls (mean \pm SD $1.20 \pm 0.73$ vs $0.80 \pm 0.39, t=2.2, p=0.016$ ) and correlated significantly with the wake time during the sleep period $(r=0.61, p=0.007)$ and all other RLS-related polysomnographic sleep variables $(p<$ 0.05) except for periodic leg movements during sleep (PLMS)/hour.

Conclusions: The primary findings introduce 2 new related dimensions to RLS: abnormalities in a major nondopaminergic neurologic system and the arousal disturbance of sleep. The strong relation of the arousal sleep disturbance to glutamate and the lack of relation to the PLMS motor features of RLS contrasts with the reverse for dopamine of a limited relation to arousal sleep disturbance but strong relation to PLMS. Understanding this dichotomy and the interaction of these 2 differing systems may be important for understanding RLS neurobiology and developing better treatments for RLS. Neurology ${ }^{\circledR}$ 2013;80:2028-2034

\section{GLOSSARY}

Cho = choline-containing compounds; $\mathbf{C r}=$ creatine/phosphocreatine; $\mathbf{G l n}=$ glutamine; Glu = glutamate; Glx = glutamine plus glutamate; ${ }^{1} \mathbf{H}$ MRS = proton magnetic resonance spectroscopy; $\mathbf{m l}=$ myo-inositol; $\mathbf{N A A}=\mathbf{N}$-acetylaspartate; $\mathbf{P L M S}=$ periodic leg movements during sleep; PSG = polysomnography; RLS = restless legs syndrome; $\mathbf{S W S}=$ slow-wave sleep; VMB $=$ ventral midbrain; WDSP = wake during the sleep period.

Restless legs syndrome (RLS) is a rest-induced, movement-responsive, mostly nocturnal, urge to move the legs commonly associated with periodic leg movements during sleep (PLMS). ${ }^{1}$ Sleep disruption is the primary factor producing most of the morbidity of moderate to severe RLS. ${ }^{2}$ Patients with RLS, however, rarely report problems with excessive daytime sleep despite total sleep times averaging less than 5.5 hours. ${ }^{3}$ Untreated subjects with RLS who have no other sleep disorders (e.g., sleep apnea) have not been found in any clinical study to have a problem falling asleep while driving nor do they show the degree of frontal lobe cognitive deficits expected from their sleep loss. ${ }^{4}$

Dopaminergic treatments provide effective treatment for RLS symptoms and dramatically reduce PLMS but in most studies fail to significantly reduce the sleep loss, arousals during sleep, ${ }^{5-7}$ and abnormal cycling alternating pattern of sleep occurring with RLS. ${ }^{8}$ Dopaminergic systems do not appear to be primary for producing the RLS sleep/wake arousal.

If not dopamine, then what produces this RLS arousal pattern? A review of potential nondopaminergic systems led to the consideration that increased glutamatergic system activation may produce the RLS arousal pattern. Thalamic abnormalities have been associated with RLS symptoms, ${ }^{9,10}$ and the thalamocortical system serves critical functions that if overactive could disrupt 
the ability to sleep in the night and day. ${ }^{11}$ This study focused on thalamic glutamatergic activity using as a surrogate measure total thalamic glutamate (Glu) and glutamine (Gln) concentration assessed by proton magnetic resonance spectroscopy $\left({ }^{1} \mathrm{H}\right.$ MRS)

METHODS Standard protocol approvals, registrations, and patient consents. The study and informed consent procedures were approved by the Johns Hopkins Institutional Review Board following the guidelines of the Declaration of Helsinki. ${ }^{12}$ All available data obtained from the eligible participants consenting to the study were included in the analyses.

Procedures. Patients eligible for this study had to be diagnosed by a Johns Hopkins RLS specialist (R.P.A. or C.J.E.) with primary RLS meeting all RLS diagnostic criteria. ${ }^{1}$ Patients with secondary causes or factors exacerbating RLS or complicating the diagnostic process were excluded. Patients with RLS had to have RLS symptoms occurring (or reported to occur when off medications) at least 6-7 days a week persisting for at least 6 months. They had to have a 5-night-average PLMS $\geq 20$ on the PAM-RL leg activity meter ${ }^{13}$ (Phillips-Respironics) after being off RLS medications for at least 7 days. Control subjects were selected to match age and sex of the patients with RLS. They were required to be free of features defining RLS, have no known first-degree relatives with RLS, be free from any significant medical or psychiatric disease and any sleep disorder (aside from RLS), and have PAM-RL PLMS $\leq 10$ /hour for 5 nights. Apnea rates determined by home screening with oral air flow or sleep laboratory polysomnography (PSG) had to be $\leq 15 /$ hour for all participants in this study. All RLS medications, all psychoactive medications (except caffeine and nicotine), and all supplements with iron were stopped at least 2 weeks prior to starting the study.

${ }^{1} \mathrm{H}$ MRS of the right thalamus was performed in the morning between 8:00 and 12:00 using a 1.5 T GE MRI scanner and the PROBE-P (PRESS) sequence. The scan parameters were $2 \times 2 \times$ $2 \mathrm{~cm}^{3}$ voxel size, 64 averages, $2,000 \mathrm{~Hz}$ sweep width, and 1,024 data points. The repetition time was 1.5 seconds and the echo time was 35 milliseconds. MRS signals were corrected for eddy currents using the unsuppressed water signal and filtered with a $2-\mathrm{Hz}$ Gaussian line-broadening function. After Fourier

Figure 1 Single voxel ${ }^{1} \mathrm{H}$ magnetic resonance spectrum of the thalamic region in patient with restless legs syndrome

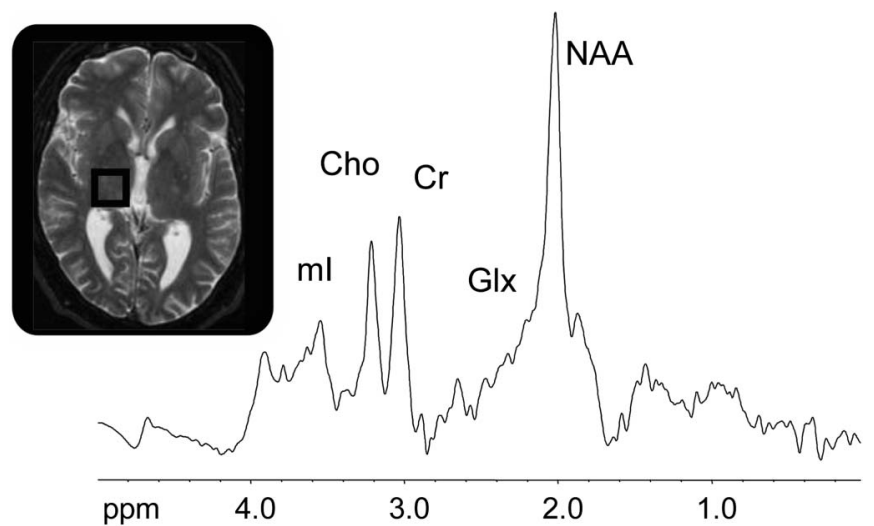

Signals of myo-inositol (ml), total choline (Cho), total creatine ( $\mathrm{Cr}$ ), glutamate and glutamine (Glx), and $N$-acetylaspartate (NAA) were evaluated. transformation, an automated zero-order phase correction was performed. The baseline correction was performed using a cubic spline routine. Signal areas of myo-inositol (mI), choline-containing compounds (Cho), creatine/phosphocreatine (Cr), Gln plus Glu (Glx), and $N$-acetylaspartate (NAA) were determined by integration in the following limits: $\mathrm{mI}$ (3.70-3.40 ppm), Cho (3.30-3.12 ppm), Cr (3.12-2.92 ppm), Glx (2.41-2.14 ppm), and NAA (2.14-1.90 $\mathrm{ppm}$ ), using an in-house program "csx2" (figure 1). MRS experts (P.B., A.H.) made all measures blinded to any knowledge about the patient or patient's diagnosis. As per standard MRS practice, metabolite signals in this study were expressed as ratios to total creatine (i. e., creatine plus phosphocreatine peak areas). The primary MRS measure for this study was the $\mathrm{Glx} / \mathrm{Cr}$ ratio. The complex Glx signal includes mostly both Glu and Gln reflecting glutamatergic activity: Glu as the available neurotransmitter, Gln as the active transport for recycling Glu. Changes in $\mathrm{Glx} / \mathrm{Cr}$ are considered to mainly reflect changes in glutamatergic metabolism.

A subgroup of subjects as part of another ongoing study had 2 consecutive nights of PSG, including a full night's PSG recording (6 hours or more) on the second night in the Hopkins Clinical Research Unit prior to the MRS. These data were used for analyses of sleep characteristics of the patients. The PSG recordings were scored to maintain consistency with our prior studies following Rechtschaffen and Kales criteria ${ }^{14}$ using C3-A2 or C2-A1 electrode derivations and PLM criteria defined by the Atlas Task Force for recording and scoring leg movements. ${ }^{15}$ Stage 3 and 4 sleep were combined as slow-wave sleep (SWS). Subjects estimated their total sleep times upon awakening at the end of their sleep period, and the average over 3 consecutive nights prior to the MRS provided the subjective measure of total sleep.

Statistical analyses. The primary measure of thalamic glutamatergic activity for this study is the MRS Glx/Cr ratio. The primary measure of RLS arousal producing wakefulness during the sleep period was selected a priori to be time spent awake during the sleep period (wake during the sleep period [WDSP]). WDSP was calculated from the second night PSG recordings as total time awake summed across the total period designated for sleep. We tested 2 primary a priori hypotheses: 1 ) the thalamic $\mathrm{Glx} / \mathrm{Cr}$ ratio will be greater for RLS than control subjects, and 2) the thalamic Glx/Cr ratio in RLS will correlate with WDSP. The first hypothesis was tested using a one-sided $t$ test and a Dixon test evaluating possible outliers. The second hypothesis was tested using a standard single-alternative regression analysis with significance set at 0.05 . All other subjective and objective sleep variables were part of secondary analyses that included comparisons between control and RLS groups and correlation to Glx/Cr ratio. Secondary analyses were tested using 2 -sided $\chi^{2}$ and $t$ tests as appropriate without correction for multiple testing for these exploratory evaluations.

Sample sizes had been set to detect effect sizes of about 0.75 and correlations with clinical parameters of about 0.6 that had been reported for iron status measurements. ${ }^{16,17}$

RESULTS Thalamic MRS data were obtained on 20 controls and 28 patients with RLS. The 2 groups were well-matched for age and sex (table 1). Mean thalamic $\mathrm{Glx} / \mathrm{Cr}$ ratio was significantly higher for patients with RLS than controls (mean \pm SD $1.20 \pm 0.73$ vs 0.80 $\pm 0.39, t=2.2$, one-tailed $p=0.016$ ) (figure 2 ). The RLS group had the 7 highest Glx/Cr values. Dixon tests indicated that the 1 or 2 highest values for patients with RLS were not significant outliers (Dixon $\mathrm{Q}=$ 0.097 for 1 and 0.37 for 2 highest values, $p>0.05^{18}$ ). 


\begin{tabular}{|llll|}
\hline Table 1 & \multicolumn{3}{l|}{$\begin{array}{l}\text { Subject variables and magnetic resonance spectroscopy for patients } \\
\text { with RLS and matching controls }\end{array}$} \\
& Controls $(\mathbf{n}=20)$ & RLS $(\mathbf{n}=28)$ & Significance \\
\hline Age, $y$, mean \pm SD & $62.6 \pm 9.3$ & $62.8 \pm 9.9$ & $t=0.1, N S$ \\
$\%$ Female & 60.0 & 53.5 & $\chi^{2}=0.2, p=0.66$ \\
Glx/Cr, mean \pm SD & $0.80 \pm 0.39$ & $1.20 \pm 0.73$ & $t=2.2, p=0.016$
\end{tabular}

Abbreviations: $\mathrm{Cr}=$ creatine/phosphocreatine; $\mathrm{Glx}=$ glutamine plus glutamate; $\mathrm{RLS}=$ restless legs syndrome. (table 2). No significant decrease in the amount of SWS and a slight, nonsignificant increase in the amount of REM sleep were found for the patients with RLS compared to the control groups. All of the sleep variables that differ between patients with RLS and controls correlated significantly with the Glx/Cr ratio except for PLMS/hour. The PLMS/hour did not significantly $(p>0.20)$ correlate with the $\mathrm{Glx} / \mathrm{Cr}$ or any of the EEG sleep variables except for a marginally nonsignificant correlation of decreasing PLMS/hour with more SWS minutes $(r=0.46, p=$ 0.055).

The other MRS measurements $(\mathrm{mI} / \mathrm{Cr}$, Cho/ $\mathrm{Cr}$, and NAA/Cr) showed no statistically significant difference between patients with RLS and controls.

DISCUSSION This study provides 2 important findings that if confirmed in further studies could significantly affect both the concepts of the underlying RLS neurobiology and our approach to evaluating and treating RLS. First, the findings support the general hypothesis of increased glutamatergic activity in RLS. If confirmed in future studies, this represents a new RLS abnormality in a major nondopaminergic neurologic system. The involvement of the thalamus indicates that the mechanism producing the arousal disrupting RLS sleep may involve increased thalamocortical activation. Whether or not other brain regions will also have increased glutamatergic activity remains to be determined.

Second, while previous studies have related iron status to RLS morbidity, ${ }^{16,19}$ these are the first data to demonstrate a relation between a neurotransmitter system and the primary morbidity of RLS, that is, sleep disruption. In particular, there are no data relating degree of dopamine abnormality to any clinical or PSG feature of RLS. These data confirm the hypothesized relation between increased $\mathrm{Glx} / \mathrm{Cr}$ levels and the degree of RLS arousal abnormality as measured by the amount of waking during the sleep period. The significant relations to $\mathrm{Glx} / \mathrm{Cr}$ occur not only for the primary measure of arousal disturbance (wake during sleep period) but also for all of the measures of sleep disruption, including the subjective estimates of total sleep times. It has been somewhat surprising to find that PLMS rates have essentially no relation to sleep fragmentation of RLS. ${ }^{20,21}$ Thus, the arousal disturbance and PLMS appear to represent somewhat different abnormalities of RLS.

Medication responses also support the concept of dual mechanisms potentially underlying the clinical abnormalities seen in RLS. On one hand, the $\alpha-2-\delta$ anticonvulsants gabapentin, pregabalin, and gabapentin enacarbil decrease Glu release and provide effective treatment for RLS. They are most effective for improving sleep with less benefit for reducing PLMS 
Table 2 Subject and night 2 polysomnography measures and Pearson correlation with thalamic $\mathrm{Glx} / \mathrm{Cr}^{\mathrm{a}}$

\begin{tabular}{|c|c|c|c|c|c|}
\hline Measure & $\begin{array}{l}\text { Controls }(n=14), \\
\text { average } \pm S D\end{array}$ & $\begin{array}{l}\text { RLS }(n=18) \\
\text { average } \pm \text { SD }\end{array}$ & $\begin{array}{l}\text { RLS vs controls } \\
t \text { test, } p^{b}\end{array}$ & $\begin{array}{l}\text { RLS correlation } \\
\text { with } \mathrm{Gl} / \mathrm{Cr}^{\mathrm{c}}\end{array}$ & Correlation, $p^{b}$ \\
\hline Age, y & $60.1 \pm 8.2$ & $62.9 \pm 10.6$ & $0.7, \mathrm{NS}$ & -0.21 & NS \\
\hline$\%$ Female & $50.0 \%$ & $55.6 \%$ & $\chi^{2}=0.98$, NS & $-^{d}$ & - \\
\hline Subject hours sleep & $7.1 \pm 0.8$ & $4.9 \pm 2.1$ & $3.5,0.002$ & -0.51 & 0.046 \\
\hline WDSPe & $48.5 \pm 15.3$ & $85.9 \pm 65.4$ & $2.1,0.045$ & +0.61 & 0.007 \\
\hline WASO & $38.6 \pm 15.2$ & $65.0 \pm 45.6$ & $2.0,0.054$ & +0.56 & 0.015 \\
\hline TST & $382.8 \pm 44.0$ & $336.0 \pm 56.4$ & $2.5,0.019$ & -0.60 & 0.008 \\
\hline Sleep efficiency, \% & $89.4 \pm 5.5$ & $78.8 \pm 14.9$ & $2.4,0.022$ & -0.67 & 0.002 \\
\hline Sleep latency ${ }^{f}$ & $9.9 \pm 5.6$ & $20.9 \pm 30.3$ & $1.3, \mathrm{NS}$ & +0.47 & 0.049 \\
\hline Stage 2 latency ${ }^{f}$ & $13.8 \pm 6.6$ & $25.2 \pm 29.1$ & $1.4, \mathrm{NS}$ & +0.42 & 0.080 \\
\hline PLMS/hour & $5.4 \pm 5.2$ & $85.2 \pm 61.9$ & $4.6,0.0001$ & +0.20 & NS \\
\hline REM latency ${ }^{f}$ & $61.5 \pm 28.6$ & $78.7 \pm 49.8$ & $1.2, \mathrm{NS}$ & +0.40 & 0.096 \\
\hline Stage REM minutes & $88.6 \pm 32.4$ & $96.0 \pm 33.1$ & 0.6, NS & -0.29 & NS \\
\hline Stage non-REM minutes & $294.2 \pm 19.9$ & $240.1 \pm 47.7$ & $4.0,0.0004$ & -0.53 & 0.023 \\
\hline Stage 1 minutes & $26.8 \pm 13.8$ & $19.5 \pm 13.5$ & $1.5, \mathrm{NS}$ & -0.12 & NS \\
\hline Stage 2 minutes & $192.9 \pm 41.5$ & $165.4 \pm 50.5$ & $1.6, \mathrm{NS}$ & -0.21 & NS \\
\hline Stages $1+2$ minutes & $219.6 \pm 46.3$ & $184.9 \pm 59.6$ & $1.8,0.082$ & -0.20 & NS \\
\hline SWS minutes & $74.6 \pm 47.3$ & $55.2 \pm 39.2$ & $1.3, N S$ & -0.34 & NS \\
\hline
\end{tabular}

Abbreviations: $\mathrm{Cr}=$ creatine/phosphocreatine; Glx = glutamine plus glutamate; NS = not significant $(p>0.10) ; \mathrm{PLMS}=$ periodic leg movements during sleep; RLS = restless legs syndrome; SWS = slow-wave sleep; TST = total sleep time; WASO = wake after sleep onset; WDSP $=$ wake minutes during sleep period.

a Sleep variables are given in minutes or percent of total sleep time.

${ }^{b} p$ Values are given for these exploratory data for $p<0.10$ for 2 -tailed testing.

c Pearson $r$, correlation with thalamic $\mathrm{Glx} / \mathrm{Cr}$ for patients with RLS only.

${ }^{\mathrm{d}}$ There were no significant sex differences in $\mathrm{Glx} / \mathrm{Cr}$ for either patients with RLS or controls.

${ }^{\text {e }}$ Total wake minutes during sleep period, includes sleep onset and wake after sleep onset. This is the designated primary outcome measure of RLS hyperarousal.

${ }^{f}$ Sleep latency is from lights out to first sleep epoch, stage 2 latency is from lights out to first stage 2 or stage 3 epoch, REM latency is from first sleep epoch to first REM epoch.

than the dopamine agents. ${ }^{22}$ The NMDA receptor antagonist ketamine had also been reported to dramatically improve sleep and reduce RLS symptoms for 2 treatment-resistant cases. ${ }^{23}$ Methadone, unlike other opioids, is effective for RLS at doses much lower than the usual analgesic dose. At the dose range used for RLS, methadone's action includes NMDA receptor antagonism. ${ }^{24-26}$ On the other hand, dopaminergic treatments dramatically reduce PLMS and in general RLS sensory symptoms but usually fail to significantly reduce the RLS arousal abnormalities increasing waking during sleep. ${ }^{5,727}$ RLS pathophysiology may involve 2 different neurotransmitter systems, dopamine and Glu, each primarily related to different clinical features of RLS. These systems interact so that treating one may indirectly alter the other but to a lesser extent and at higher doses. Thus, there is some reduction in PLMS with $\alpha-2-\delta$ drugs but it is less consistent and of a smaller magnitude than with dopaminergic drugs. Conversely, there may be some decrease in waking during sleep with dopaminergics, but again, it is less consistent and of a smaller magnitude than with $\alpha-2-\delta$ drugs. Confirming this concept in future studies may be important for evaluating and treating RLS. A focus on only one of these aspects or treatments may lead to either excessive doses or less than optimal patient care.

The nature of the RLS arousal disturbance is itself interesting in relation to the thalamic MRS data. The 4 studies comparing EEG-determined sleep of patients with RLS to matched controls have all shown significantly increased awakening and decreased sleep efficiency. ${ }^{20,28-30}$ All but on ${ }^{28}$ report significantly decreased total sleep time. Two of the 4 studies report significantly decreased stage 2 sleep. ${ }^{20,31}$ Only one study reported significantly decreased REM sleep ${ }^{20}$ and then by only a small amount $(0.3 \%$ of sleep period) with minimal statistical significance not corrected for multiple comparisons. None of these studies reported significant differences between RLS and controls for SWS. The data in this study (table 2) show the same pattern of increased wake affecting mostly stage 1 and 2 sleep with no significant effect on either SWS or REM sleep. Considering all of these 
Figure 3 Linear regressions of wake during sleep, total sleep time, and subjective estimate of sleep time vs right thalamic $\mathrm{Glx} / \mathrm{Cr}$

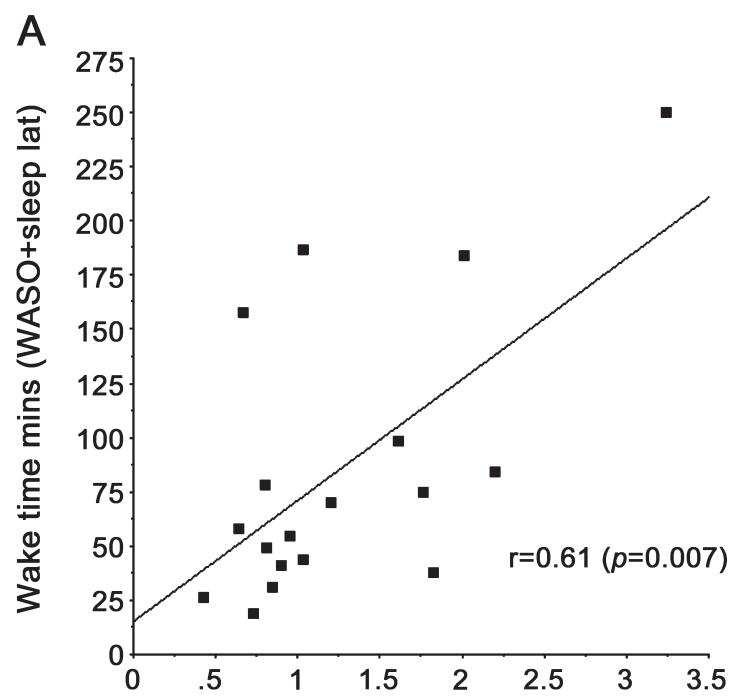

B
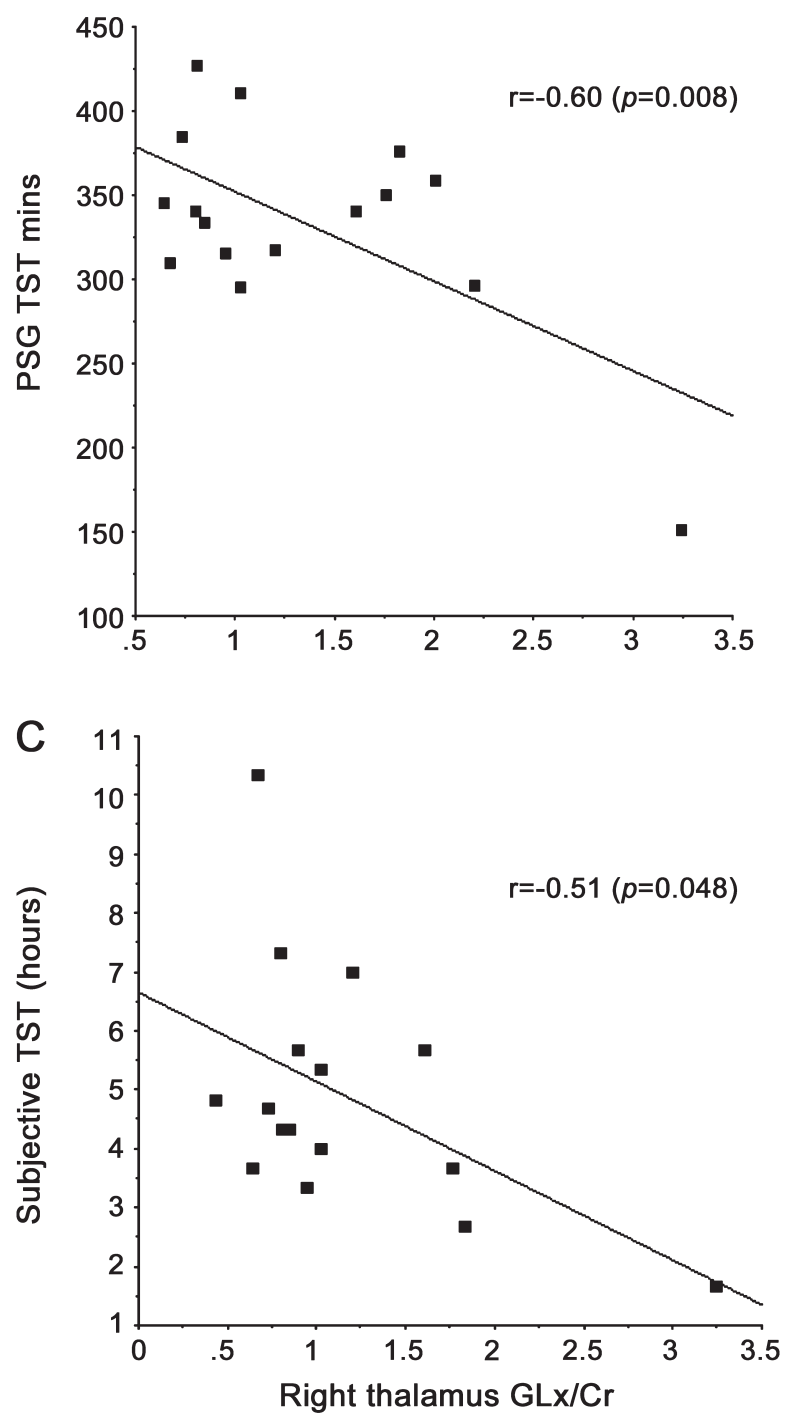

(A) Wake during sleep, (B) total sleep time (TST), (C) subjective estimate of sleep time. $\mathrm{Cr}=$ creatine/ phosphocreatine; $\mathrm{Glx}=$ glutamine plus glutamate; $\mathrm{PSG}$ = polysomnography; WASO = wake after sleep onset. studies, it appears that RLS-related sleep disturbance is expressed by increased waking during stage 2 sleep and to lesser extent other non-REM sleep with little effect on REM. It is striking that in the current study the thalamic $\mathrm{Glx} / \mathrm{Cr}$ correlates significantly with all of the sleep characteristics that show significant differences between RLS and controls except for PLMS (table 2). The hyperpolarization of thalamocortical neurons provides a primary regulation for sleep spindle generation and for reduction of sensory inputs enabling cortical sleep. ${ }^{11}$ Increased thalamocortical excitation would therefore be expected to produce both the increased wake time and decreased stage 2 sleep seen in patients with RLS. Moreover, REM sleep represents a more activated brain state that could be much less affected if at all by increased thalamic excitatory activity. The relative sparing of SWS may reflect somewhat different neurobiological mechanisms supporting SWS vs stage 2 sleep. Thus, the increased thalamic glutamatergic activity indicated in this study could explain the sleep disruption characteristic of RLS.

What is not clear from these data is whether the increased glutamatergic activity causes or results from the sleep disruption at night. There is one study of primary insomnia that provides relevant neurochemical data. The primary insomnia patients had sleep disruption close to that seen in our patients with RLS, i.e., averages for the insomnia vs RLS patients with sleep efficiency $80.0 \%$ vs $78.8 \%$, wake after sleep onset 64.3 vs 65.0 minutes, sleep latency 23.2 vs 20.9 minutes, and total sleep time 384.3 vs 336.0 minutes, respectively. The PLMS/hour were far fewer for primary insomnia than RLS (averages 2.6 vs 85.2 , respectively), but PLMS do not correlate in any way with the degree of sleep disturbance in the RLS data. MRS from a $4 \mathrm{~T}$ MRI showed these primary insomnia patients compared to matched controls had significantly decreased GABA but no change in Glx. ${ }^{32}$ If it were the sleep disruption itself that produced the Glx increase in RLS, then at least some increase in Glx for primary insomnia would be expected, contrary to the report here. It should be noted, however, that this is only one study, and primary insomnia is a complicated disorder involving multiple factors that could disrupt sleep. A better control for future studies would be experimentally sleep-deprived controls matched for sleep times and arousal during sleep with that experienced by patients with RLS. The insomnia study, however, provides us with the best available comparison data and provides some assurance that the Glx differences in this study do not occur with the sleep disruption alone.

Finally, if there is a glutamatergic pathology in RLS, the question then becomes what could be causing this abnormality? Compromised brain iron homeostasis is a well-established feature of RLS documented by multiple studies indicating reduced iron concentration in select brain regions ${ }^{33-35}$ that particularly include 
decreased H-ferritin in the substantia nigra. ${ }^{36,37}$ Two studies have used MRS to evaluate Glu levels in animals with compromised brain iron status. Gestational and lactational dietary iron deficiency produced a decrease in brain iron and a significant increase in rat striatal Glu. ${ }^{38}$ Similarly, mice with a genetic H-ferritin deficiency producing a condition similar to the H-ferritin deficiency in patients with $\operatorname{RLS}^{36,37}$ also showed increased $\mathrm{Glu}^{39}$ present in several brain regions. Moreover, quantitative trait locus mapping of genetic factors related to murine ventral midbrain (VMB) iron identified a relation between VMB iron status and the glial high-affinity Glu transporter gene (Glt-1). Glt-1 expression showed a strong negative correlation with $\mathrm{VMB}$ iron. ${ }^{40}$ While this study did not measure protein, it could, nonetheless, indicate that iron deficiency occurs with increased glial uptake of Glu, consistent with the primary hypothesis of this project of increased glutamatergic activity in RLS.

There are 3 limitations to this study. First, despite having theoretically based primary hypotheses, this has to be considered as an initial study of MRS and RLS. The results need to be confirmed in another adequate sample size study. Second, the patients had severe RLS with almost daily symptoms and significant PLMS and sleep disturbance. It is unclear if the results generalize to less severe RLS. Third, the MRS measures of Glx in this study are a combination of Glu and Gln with possibly a relatively small amount of GABA. Given the close metabolic relation between Glu and GABA, it is possible that GABA might also be increased, although this would not seem likely to produce sleep loss observed in RLS. Glu also serves multiple functions in the brain, and changes in Glu levels themselves are hard to interpret. It also cannot be discounted that some of the change in the $\mathrm{Glx} / \mathrm{Cr}$ ratio may be due to changes in $\mathrm{Cr}$ rather than Glx. If this occurred, however, it would be expected to also produce differences in the other MRS measures, which were not found. The finding in this study should be further evaluated with MRS studies using higher magnetic field strengths and quantification methods that provide separate Glu, Gln, and GABA measurements.

Overall, these data provide a consistent pattern indicating a significant increase in thalamic glutamatergic activity that could produce hyperarousal in patients with RLS associated with increased waking during sleep, mostly affecting non-REM sleep and not related to PLMS. This RLS hyperarousal could also reduce the effects of the chronic sleep loss with RLS during the daytime. The Glu and dopamine abnormalities may combine and interact to produce the full range of RLS symptoms, i.e., dopamine related more to sensory symptoms and PLMS, Glu related more to the RLS hyperarousal with sleep disruption. The relative clinical and biological importance and interaction of these systems for RLS and the significance of the putative RLS hyperarousal remain to be determined.

\section{AUTHOR CONTRIBUTIONS}

Dr. Richard P. Allen: concept and design of study, participant evaluations, data analyses, wrote first and final drafts of article. Dr. Christopher J. Earley: concept and design of study, participant evaluations, reviewed data analyses, edited the drafts of the article. Dr. Alena Horská and Dr. Peter Barker: responsible for all MRI scans and the data reduction for spectroscopy, edited the drafts of the article.

\section{STUDY FUNDING}

Supported in part by NIH grants R01 NS075184, P10-AG21190, NS044862, and M01RR02719.

\section{DISCLOSURE}

R.P. Allen has received honoraria serving on advisory boards from IMPAX, Pfizer, and UCB, and honoraria for scientific lectures, consultancy, and research support from UCB, GSK, Pfizer, and pharmacosmos. P.B. Barker, A. Horská, and C.J. Earley report no disclosures. Go to Neurology.org for full disclosures.

Received November 5, 2012. Accepted in final form January 24, 2013.

\section{REFERENCES}

1. Allen RP, Picchietti D, Hening WA, Trenkwalder C, Walters AS, Montplaisir J. Restless legs syndrome: diagnostic criteria, special considerations, and epidemiology: a report from the restless legs syndrome diagnosis and epidemiology workshop at the National Institutes of Health. Sleep Med 2003;4:101-119.

2. Kushida CA, Allen RP, Atkinson MJ. Modeling the causal relationships between symptoms associated with restless legs syndrome and the patient-reported impact of RLS. Sleep Med 2004;5:485-488.

3. Allen RP, Stillman P, Myers AJ. Physician-diagnosed restless legs syndrome in a large sample of primary medical care patients in western Europe: prevalence and characteristics. Sleep Med 2010;11:31-37.

4. Gamaldo CE, Benbrook AR, Allen RP, Oguntimein O, Earley CJ. A further evaluation of the cognitive deficits associated with restless legs syndrome (RLS). Sleep Med 2008;9:500-505.

5. Oertel WH, Benes H, Garcia-Borreguero D, et al. Rotigotine transdermal patch in moderate to severe idiopathic restless legs syndrome: a randomized, placebo-controlled polysomnographic study. Sleep Med 2010;11:848-856.

6. Jama L, Hirvonen K, Partinen M, et al. A dose-ranging study of pramipexole for the symptomatic treatment of restless legs syndrome: polysomnographic evaluation of periodic leg movements and sleep disturbance. Sleep Med 2009; 10:630-636.

7. Allen R, Becker PM, Bogan R, et al. Ropinirole decreases periodic leg movements and improves sleep parameters in patients with restless legs syndrome. Sleep 2004;27:907-914.

8. Ferri R, Manconi M, Arico D, et al. Acute dopamine-agonist treatment in restless legs syndrome: effects on sleep architecture and NREM sleep instability. Sleep 2010;33:793-800.

9. Walters AS, Ondo WG, Zhu W, Le W. Does the endogenous opiate system play a role in the restless legs syndrome? A pilot post-mortem study. J Neurol Sci 2009;279:62-65.

10. Bucher S, Seelos K, Oertel W, Reiser M, Trenkwalder C. Cerebral generators involved in the pathogenesis of the restless legs syndrome. Ann Neurol 1997;41:639-645. 
11. Coulon P, Budde T, Pape HC. The sleep relay-the role of the thalamus in central and decentral sleep regulation. Pflugers Arch 2012;463:53-71.

12. Lynoe $\mathrm{N}$, Sandlund M, Dahlqvist G, Jacobsson L. Informed consent: study of quality of information given to participants in a clinical trial. BMJ 1991;303:610-613.

13. Sforza E, Johannes M, Claudio B. The PAM-RL ambulatory device for detection of periodic leg movements: a validation study. Sleep Med 2005;6:407-413.

14. Rechtschaffen A, Kales A, eds. A Manual of Standardized Terminology, Techniques and Scoring System for Sleep Stages of Human Subjects. Los Angeles: UCLA Brain Information Service/Brain Research Institute; 1968.

15. Atlas Task Force of the American Sleep Disorders Association. Recording and scoring leg movements. Sleep 1993; 16:748-759.

16. Allen RP, Barker PB, Wehrl F, Song HK, Earley CJ. MRI measurement of brain iron in patients with restless legs syndrome. Neurology 2001;56:263-265.

17. Earley CJ, Barker PB, Horska A, Allen RP. MRI-determined regional brain iron concentrations in early- and lateonset restless legs syndrome. Sleep Med 2006;7:459-461.

18. Dean R, Dixon WJ. Simplified statistics for small numbers of observations. Anal Chem 1951;23:636-638.

19. Sun ER, Chen CA, Ho G, Earley CJ, Allen RP. Iron and the restless legs syndrome. Sleep 1998;21:371-377.

20. Hornyak M, Feige B, Voderholzer U, Philipsen A, Riemann D. Polysomnography findings in patients with restless legs syndrome and in healthy controls: a comparative observational study. Sleep 2007;30:861-865.

21. Montplaisir J, Boucher S, Poirier G, Lavigne G, Lapierre O, Lesperance P. Clinical, polysomnographic, and genetic characteristics of restless legs syndrome: a study of 133 patients diagnosed with new standard criteria. Mov Disord 1997;12:61-65.

22. Winkelman JW, Bogan RK, Schmidt MH, Hudson JD, Derossett SE, Hill-Zabala CE. Randomized polysomnography study of gabapentin enacarbil in subjects with restless legs syndrome. Mov Disord 2011;26:2065-2072.

23. Kapur N, Friedman R. Oral ketamine: a promising treatment for restless legs syndrome. Anesth Analg 2002;94: 1558-1559.

24. Davis AM, Inturrisi CE. d-Methadone blocks morphine tolerance and N-methyl-D-aspartate-induced hyperalgesia. J Pharmacol Exp Ther 1999;289:1048-1053.

25. Ebert B, Andersen S, Krogsgaard-Larsen P. Ketobemidone, methadone and pethidine are non-competitive $\mathrm{N}$-methyl-D-aspartate (NMDA) antagonists in the rat cortex and spinal cord. Neurosci Lett 1995;187:165-168.

26. Ebert B, Thorkildsen C, Andersen S, Christrup LL, Hjeds H. Opioid analgesics as noncompetitive N-methyl-
D-aspartate (NMDA) antagonists. Biochem Pharmacol 1998;56:553-559.

27. Partinen M, Hirvonen K, Jama L, et al. Efficacy and safety of pramipexole in idiopathic restless legs syndrome: a polysomnographic dose-finding study-the PRELUDE study. Sleep Med 2006;7:407-414.

28. Montplaisir J, Boucher S, Nicolas A, et al. Immobilization tests and periodic leg movements in sleep for the diagnosis of restless leg syndrome. Mov Disord 1998;13:324-329.

29. Saletu B, Gruber G, Saletu M, et al. Sleep laboratory studies in restless legs syndrome patients as compared with normals and acute effects of ropinirole: 1: findings on objective and subjective sleep and awakening quality. Neuropsychobiology 2000;41:181-189.

30. Garcia-Borreguero D, Larrosa O, Granizo JJ, de la Llave Y, Hening WA. Circadian variation in neuroendocrine response to $\mathrm{L}$-dopa in patients with restless legs syndrome. Sleep 2004;27:669-673.

31. Saletu M, Anderer P, Saletu B, et al. Sleep laboratory studies in restless legs syndrome patients as compared with normals and acute effects of ropinirole: 2: findings on periodic leg movements, arousals and respiratory variables. Neuropsychobiology 2000;41:190-199.

32. Winkelman JW, Buxton OM, Jensen JE, et al. Reduced brain GABA in primary insomnia: preliminary data from $4 \mathrm{~T}$ proton magnetic resonance spectroscopy ( $\left.{ }^{1} \mathrm{H}-\mathrm{MRS}\right)$. Sleep 2008;31:1499-1506.

33. Allen R. Dopamine and iron in the pathophysiology of restless legs syndrome (RLS). Sleep Med 2004;5:385-391.

34. Allen RP, Earley CJ. The role of iron in restless legs syndrome. Mov Disord 2007;22:S440-S448.

35. Schmidauer C, Sojer M, Seppi K, et al. Transcranial ultrasound shows nigral hypoechogenicity in restless legs syndrome. Ann Neurol 2005;58:630-634.

36. Connor JR, Boyer PJ, Menzies SL, Dellinger B, Allen RP, Earley CJ. Neuropathological examination suggests impaired brain iron acquisition in restless legs syndrome. Neurology 2003;61:304-309.

37. Connor JR, Wang XS, Patton SM, et al. Decreased transferrin receptor expression by neuromelanin cells in restless legs syndrome. Neurology 2004;62:1563-1567.

38. Ward KL, Tkac I, Jing Y, et al. Gestational and lactational iron deficiency alters the developing striatal metabolome and associated behaviors in young rats. J Nutr 2007;137: 1043-1049.

39. Ill AM, Mitchell TR, Neely EB, Connor JR. Metabolic analysis of mouse brains that have compromised iron storage. Metab Brain Dis 2006;21:77-87.

40. Jellen LC, Unger EL, Lu L, et al. Systems genetic analysis of the effects of iron deficiency in mouse brain. Neurogenetics 2012;13:147-157. 